\title{
Method for Real-Time Abnormal State Detection of a Distribution Network Based on Maximum and Minimum Eigenvalues
}

\author{
Keyan Liu, ${ }^{1}$ Kaiyuan He, ${ }^{1}$ Huanna Niu, ${ }^{2}$ Yuzhu Wang, ${ }^{2}$ and Jingxiang Zhao ${ }^{2}$ \\ ${ }^{1}$ China Electric Power Research Institute, Beijing, China \\ ${ }^{2}$ College of Information and Electric Engineering, China Agricultural University, Beijing, China \\ Correspondence should be addressed to Huanna Niu; nhn@cau.edu.cn
}

Received 10 March 2017; Accepted 14 May 2017; Published 14 June 2017

Academic Editor: Emilio Insfran

Copyright (C) 2017 Keyan Liu et al. This is an open access article distributed under the Creative Commons Attribution License, which permits unrestricted use, distribution, and reproduction in any medium, provided the original work is properly cited.

The state analysis method of a traditional distribution network operation is strictly dependent on the physical model of itself, but it varies as the geography changes, and it is difficult to find the abnormal state of a district network on real-time, especially the sudden change caused by the distributed energy and EV load. So, a method of the abnormal state detecting for the distribution network is proposed based on the maximum and minimum eigenvalues. Firstly, a high-dimensional random matrix is established by the big data from the distribution network management system to take abnormal state detection through a real-time sliding window. Then, the maximum and minimum eigenvalues of the distribution network are gained by calculating the sample covariance matrix of the random matrix and determining the maximum and minimum eigenvalues of the latter matrix. Finally, an 1177-node testing system was taken as an example, and the simulation results showed that the proposed method could detect the abnormal state in real-time without depending on the physical model and fault type of the grid.

\section{Introduction}

The state analysis of a traditional distribution network operation that uses a physical model to establish the mathematical model and then carries on the numerical calculation has obtained very good application effects. However, a distribution network is a complex nonlinear network in which there is a coupling correlation between the various components. The mechanism of the abnormal situation of the distribution network is also more complex. The traditional method varies as the geography changes, and it is difficult to find the abnormal state of a district network on real-time, especially the sudden change caused by the distributed energy and EV load. The establishment of a physical model is generally based on certain assumptions and simplifications, and their applicability in the power grid is poor.

Distribution network operation status analysis has always been an important research topic. The detection of the abnormal state of the distribution network can provide an important basis for the operation of the relevant staff. Literature [1] proposed a method of operation situational awareness under steady state based on dynamic power flow for ADN examining grid's operation status in time-changing scenarios. Literature [2] proposed a strategy of operational status recognition based on FCM (Fuzzy C-Mean) and ANFIS (Adaptive Network Fuzzy Inference System) which constructs a kind of classifier based on ANFIS for the hierarchical fuzzy inference system and applies the FCM classification method to optimally initialize its parameters. Literature [3] proposed a method using the change in phase current unbalance due to the distributed generator operation. These methods still rely on the physical model to analyze the grid operating status and did not make full use of historical data and real-time data.

Big data technology is a new concept that has attracted increasing attention in recent years. It refers to a technical 
system or technology architecture that can capture, discover, and analyze high-volume and complex data [4-6]. Big data technology shows that the data are not independent but rather interrelated with mutual influences and more complex relationships. For example, meteorological conditions and socioeconomic situations affect a user's electricity consumption, electricity consumption data affects electricity market transactions, electricity market data provides the basis for decision-making in the relevant public service sector, and the Geographic Information System (GIS) data of the power enterprise must use the municipal planning data as a reference [7-10]. The major advantage of large data technology in power system applications is that it can be separated from the physical model to a certain extent. The relationship between data mining and grid operation can be analyzed from a datadriven perspective that can be used to solve the practical problems of power systems, such as the reactive power optimization problems [11-13]. The maximum-minimum eigenvalue (MME) algorithm was originally proposed by the Institute for Infocomm Research in 2007 and was initially applied to signal detection in the field of cognitive radio, that is, to perceive user's signals in a radio network. Based on the random matrix theory (RMT), the algorithm is used to process the signal data received by multiple antennas. The MME ratio is obtained to determine whether a received signal is an independent and identically distributed noise signal or the target user's signal. The algorithm is characterized by the use of global data and by sensing and detecting weak signals [14].

Based on the RMT, this paper uses the MME method to analyze the operating state of the power system in realtime and to accurately detect the abnormal time. In this paper, the RMT is introduced, and the detection method of maximum and minimum eigenvalue anomalies is proposed based on the stochastic matrix theory. Finally, the feasibility and correctness of the proposed algorithm are verified using a system with 1177 nodes. The results showed that the MME method had a good effect on application in the abnormal state detection of a power network, which provides a new idea for the application of large data real-time analysis technology in power network operation analysis.

\section{High-Dimensional Random Matrix Modeling of Big Data in Abnormal State Detection of a Distribution Network}

It is assumed that the distribution network system of $M$-node $(M \geq 1)$ network has a real-time measurement data device. $i_{\text {th }}$ measured data $x_{i}(t) \quad(i=1, \ldots, m, t=1,2, \ldots$ is the time series for the measurement) is a complex number, which can be a load-complex vector constituted by the active/reactive power measurement data or a voltage-complex vector for each node or branch power. Distribution network operation database is formed by massive data collected by distribution network measurement data. The sample data of a certain type electricity extracted from the operation database generate

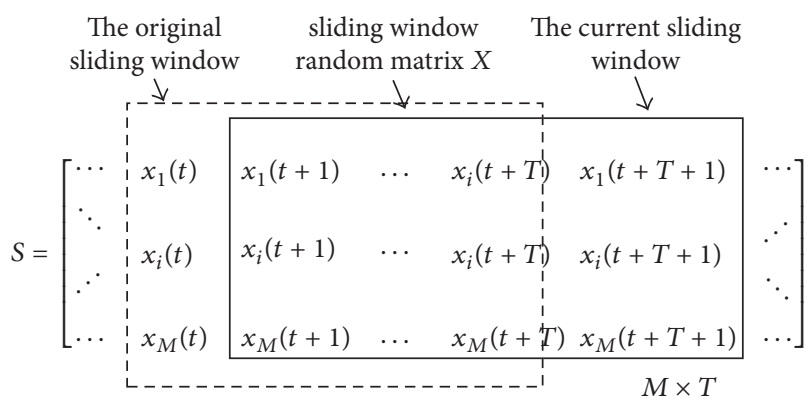

FIGURE 1: Sliding window random matrix diagram.

a high-dimensional sampling random matrix $\mathbf{S}$ of the following equation:

$$
\mathbf{S}=\left[\begin{array}{ccccc}
x_{1}(1) & \cdots & x_{1}(t) & \ldots & \cdots \\
& \ddots & & . & \\
& & x_{i}(t) & & \\
& \cdot & & \ddots & \\
x_{M}(1) & & x_{M}(t) & \ldots & \ldots
\end{array}\right] .
$$

In order to carry out the abnormal state detection, $M \times$ $T$ sliding window random matrix $\mathbf{X}$ is separated from the sampling random matrix equation (1) by sliding window method. The sliding window random matrix model is shown in Figure 1.

The first column on the right side of sliding window is the current data. Window width of the sliding window is $T$ and the sliding step $t_{\mathrm{sw}}$ is the same as the time interval between the adjacent two columns of data. Therefore, when the sliding window moves in chronological order by one step, the data of the first column on the left side of the sliding window random matrix leaves and the newly acquired data enters the first column on the right side of the sliding window random matrix. This paper constructs a simulation platform of sliding window random matrix, and the structure of the platform is shown in Figure 2.

\section{Maximum-Minimum Eigenvalue (MME) Method}

3.1. Data Acquisition and Normalization Processing. In the first step, data are acquired by the sliding window, and the new measurement data are input into the rightmost column vector $\mathbf{x}(\mathbf{T})$ in the random matrix $\mathbf{S}$ of the sliding window in the new sampling period. Next, the normalized matrix $\mathbf{X}_{\mathbf{n}}=\left(x_{i j}\right)_{m \times n}$ is obtained by normalizing the sliding window matrix $\mathbf{X}_{M \times T}$ according to (2). The expectation of the matrix is zero, and the variance is 1 .

$$
x_{i j}=\left[x_{i}(j)-\mu_{\left(\widehat{x}_{i}\right)}\right] \frac{\sigma_{\left(\tilde{x}_{i}\right)}}{\sigma_{\left(\widehat{x}_{i}\right)}}+\mu_{\left(\widetilde{x}_{i}\right)},
$$

where $\mu_{\left(\widehat{x}_{i}\right)}$ and $\sigma_{\left(\widehat{x}_{i}\right)}$ are the expected value and variance of each row before normalizing the matrix, respectively, and $\mu_{\left(\widetilde{x}_{i}\right)}$ and $\sigma_{\left(\widetilde{x}_{i}\right)}$ are the expected value and variance of each row of the normalized matrix, respectively. 


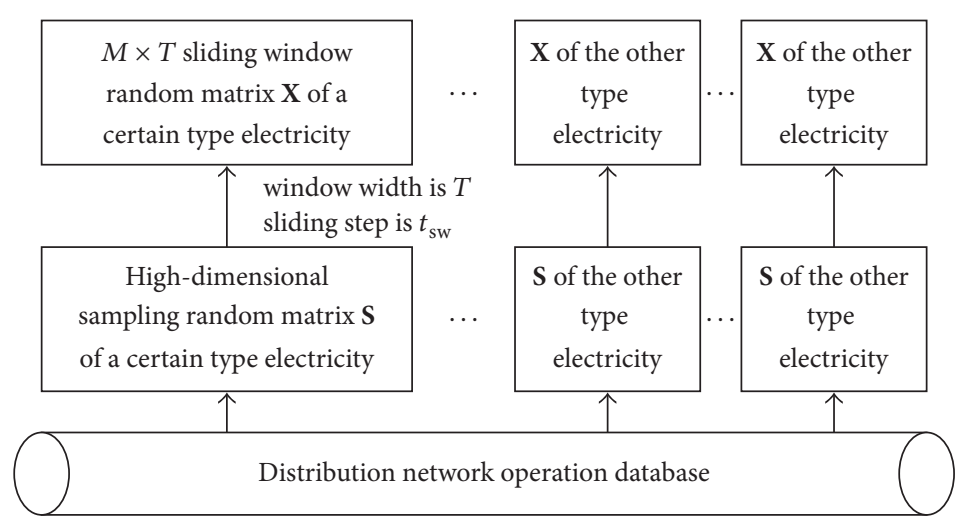

FIGURE 2: Schematic diagram of sliding window random matrix simulation platform.

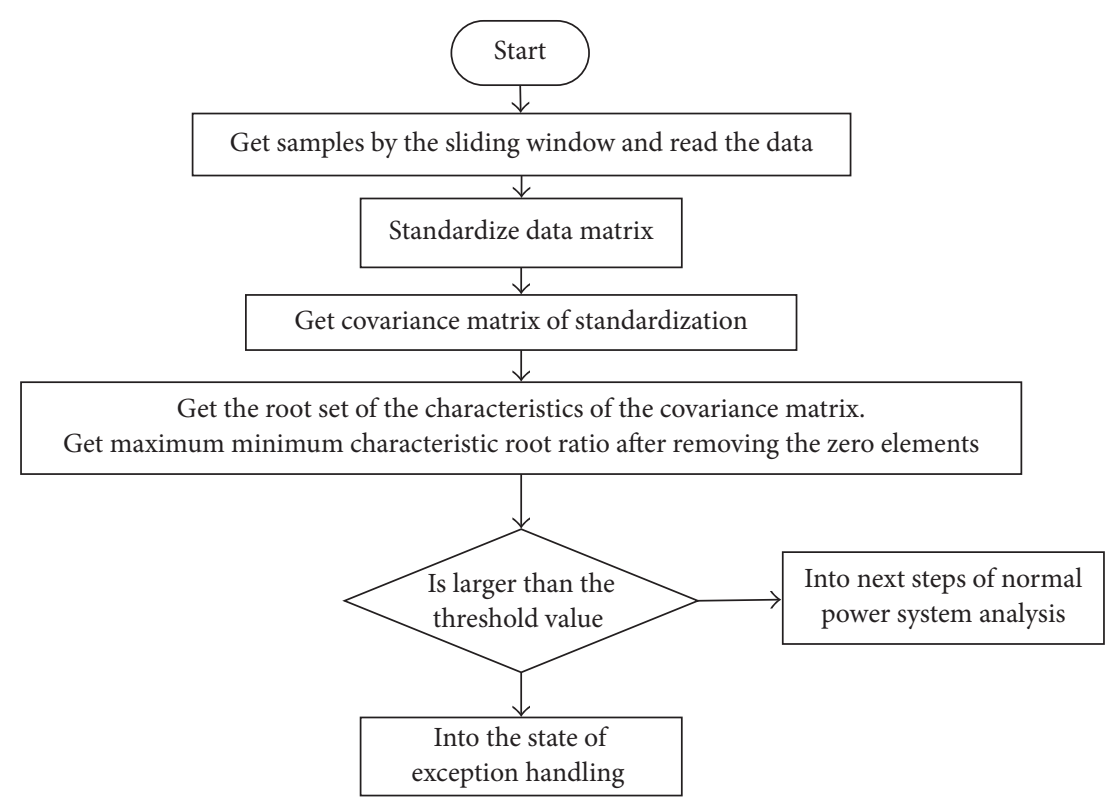

FIGURE 3: Flow chart of abnormal state detection based on the maximum-minimum eigenvalue (MME) method.

3.2. The Acquisition of the Sample Covariance Matrix. The sample covariance matrix of the measured data is obtained by the sample covariance matrix $\mathbf{R}(T)$ :

$$
\mathbf{R}(T)=\frac{1}{T} \sum_{t=1}^{T} \widehat{x}(t) \widehat{x}^{*}(t),
$$

where $*$ denotes a complex conjugate transpose and $\widehat{x}(t)$ denotes $i_{\text {th }}$ row of $X_{n}$.

3.3. Obtaining the MME Ratio $\lambda$ of the Covariance Matrix. After calculating the eigenvalues of the sample covariance matrix $\mathbf{R}(T)$, the characteristic root equaling 0 is the first to be removed. Then, the ratio $\lambda$ is calculated, which is the criterion for determining whether there is an abnormal condition [1517].

$$
\lambda=\frac{\hat{\lambda}_{\max }}{\widehat{\lambda}_{\min }} .
$$

According to the ratio $\lambda$ and the magnitude of the empirical thresholds, it is possible to know whether there are any abnormalities in the current sampling time. A flow chart of this process is shown in Figure 3.

\section{Examples}

To verify the effectiveness of the MME method, the following simulation experiments were carried out.

The system is based on an 1177-node system of a regional distribution network with 1176 branches. The schematic diagram of the system is shown in Figure 4 . The sampling time interval was $1 \mathrm{~s}$. The sliding width $T$ of the random matrix was 200. According to the actual situation of the distribution network's abnormal state, simulation of the four typical abnormal states of a single-phase short-circuit, namely, a three-phase short circuit, load mutation, and a circuit break scene, were carried out, in which setting the abnormal scene 


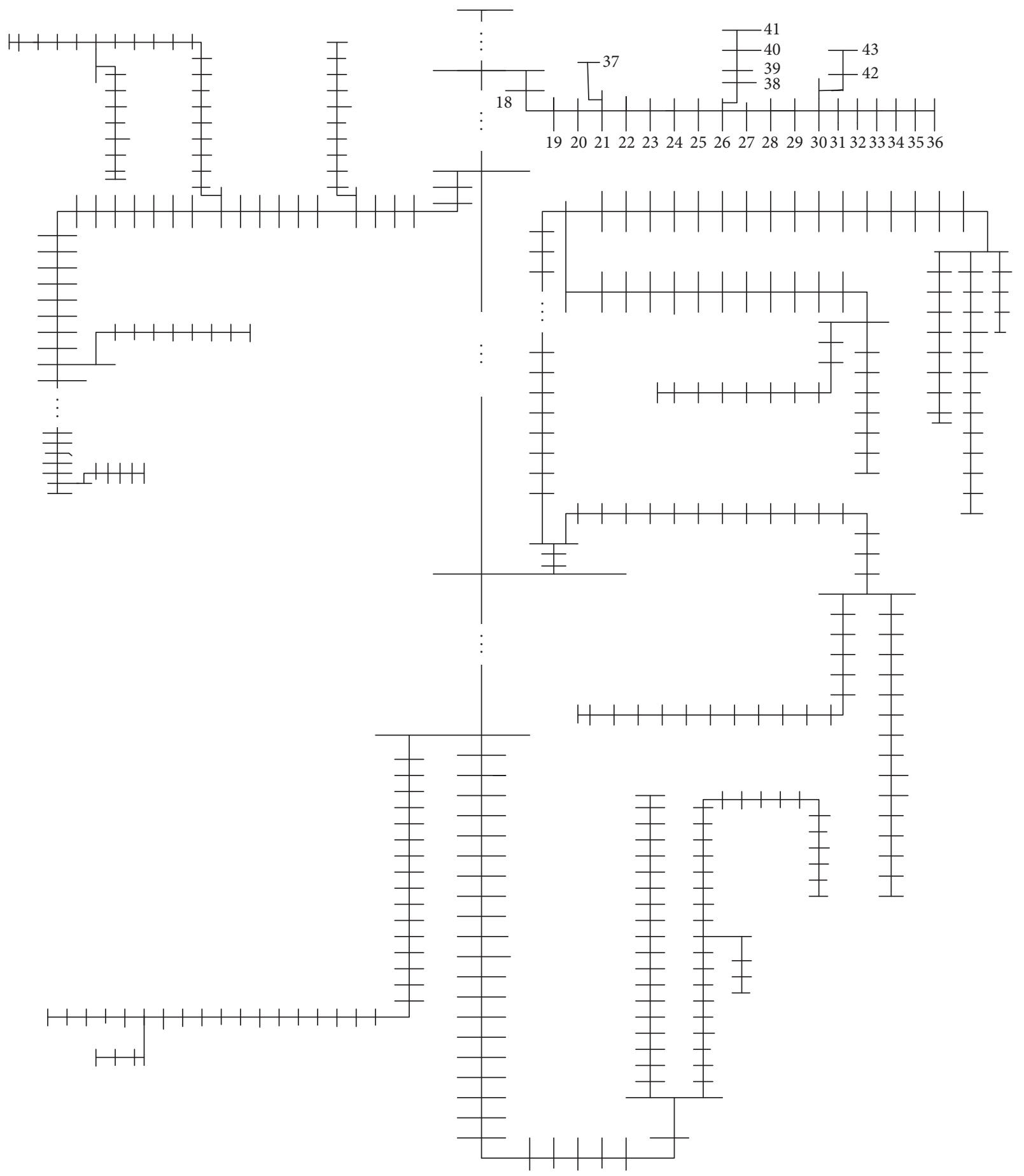

FIGURE 4: Schematic diagram of a regional 1177-node system.

occurred in a branch of the 1177-node system area (Figure 5). The maximum and minimum eigenvalue algorithms were used to detect anomaly states in the four typical anomaly scenarios. The results of detection and identification were analyzed as follows.

4.1. Single-Phase Ground Fault State Identification. In the case of a single-phase-to-ground fault, the protection of the circuit breaker does not operate at the beginning of the line. The line can continue to run for about 1 to $2 \mathrm{~h}$ in the presence of the fault, but the phase voltage drops to zero at the ground point and its downstream nodes. The nonfault phase voltage rises to a line voltage of 1.7 times.

According to the physical phenomena in the case of a single-phase-to-ground fault, the simulation data scenario is as follows. 


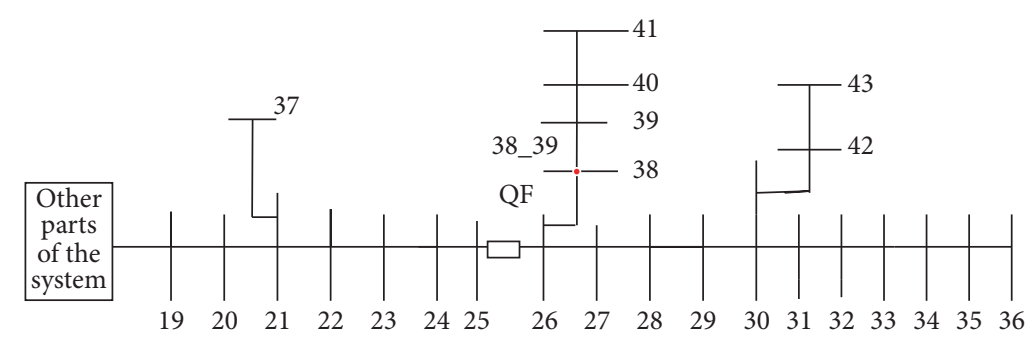

FIGURE 5: Sketch map of abnormal branch of the 1177-node system in a certain area.

It is assumed that if a single-phase ground fault occurs in phase A on branch 26_38 between nodes 26 and 38, the protection of QF does not work. The voltage of phase A is reduced to 0 , and the voltage of nonfault phases $B$ and $C$ is increased 1.7 times, and the voltage of other nodes does not change much apart from its downstream nodes in the tidal flow directions $38,39,40$, and 41 .

A node voltage matrix was used as the pending matrix for the sliding window sample. The nodal voltage matrix of the system can be expressed as follows:

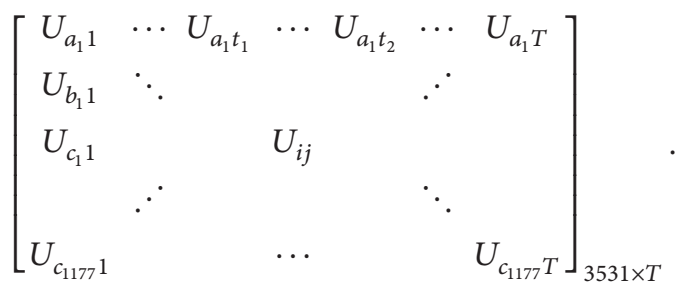

In type (5), $U_{a_{1} 1}, U_{b_{1} 1}$, and $U_{c_{1} 1}$ represent the three-phase voltage value of node 1 at the first instant, respectively, and $U_{i j}$ represents the voltage value of one phase at node $j$ at time $j$.

The detection results are shown in Figure 6, where the blue line is the MME value of the random matrix of the detected window, that is, the maximum and minimum eigenvalue ratios $\lambda$; the green dotted line represents the actual measurement, and the red line represents the threshold. The threshold is taken from the experience threshold obtained from the data when the power system runs normally.

As shown in Figure 6, at $350 \mathrm{~s}$, the branch 26_38 has a single-phase ground fault, and the MME value suddenly increases and exceeds the threshold. The MME curve appears to be significantly spiked. The second spike appears at $550 \mathrm{~s}$, that is, $200 \mathrm{~s}$ after the window width. Between the two spikes of the curve, the MME curve exhibits random fluctuations. When the sliding window continues to slide, in the first column of abnormal data into the sliding window and the last column of normal data away from the sliding window, the MME value changes the most.

\subsection{Three-Phase Short-Circuit Abnormal State Identification.} In the case of a three-phase short-circuit fault, the circuit breaker at the beginning of the line will cut off the part after the circuit breaker, including the faulty node. After the line breaker, the three-phase voltage of the node and its downstream node is reduced to zero.

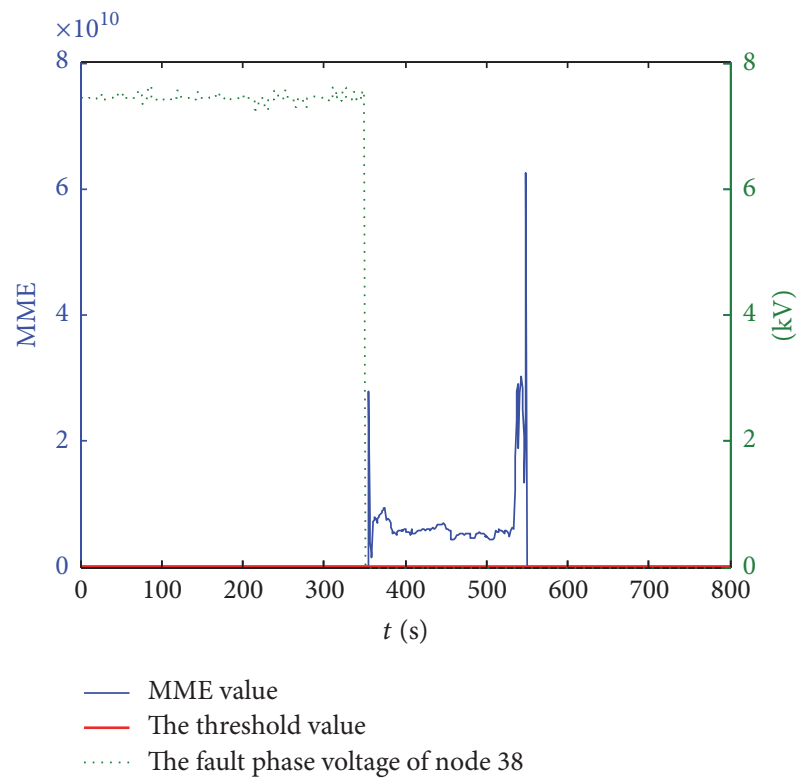

FIGURE 6: Abnormal state detection of single-phase ground fault.

According to the physical phenomena in the case of a three-phase short-circuit fault, the simulation data scenario is as follows.

It is assumed that if the three-phase short-circuit fault occurs at the branch 26 38 between the nodes 26 and 38, the protection of the circuit breaker QF between the nodes 25 and 26 is immediately activated. The three-phase voltage drops to 0 after the fault occurs, including nodes 26 to 36 and 38 to 43 , and the voltage of other nodes does not change much.

The MME curve of the three-phase short-circuit fault (Figure 7) was similar to that of the single-phase ground fault. The data matrix adopted the node voltage matrix, which can also be expressed as (4). The fault occurs at $400 \mathrm{~s}$, the MME value rises at 400 and $600 \mathrm{~s}$, and the time interval of one window is $200 \mathrm{~s}$. In this way, the time of occurrence of the three-phase short-circuit fault can be accurately detected.

4.3. Disconnected Status Abnormal State Identification. In case of a circuit break failure, the protection of the circuit breaker at the first of the line does not work, and the line can continue to run. However, when a circuit break failure happens in a branch between two nodes, the branch power 


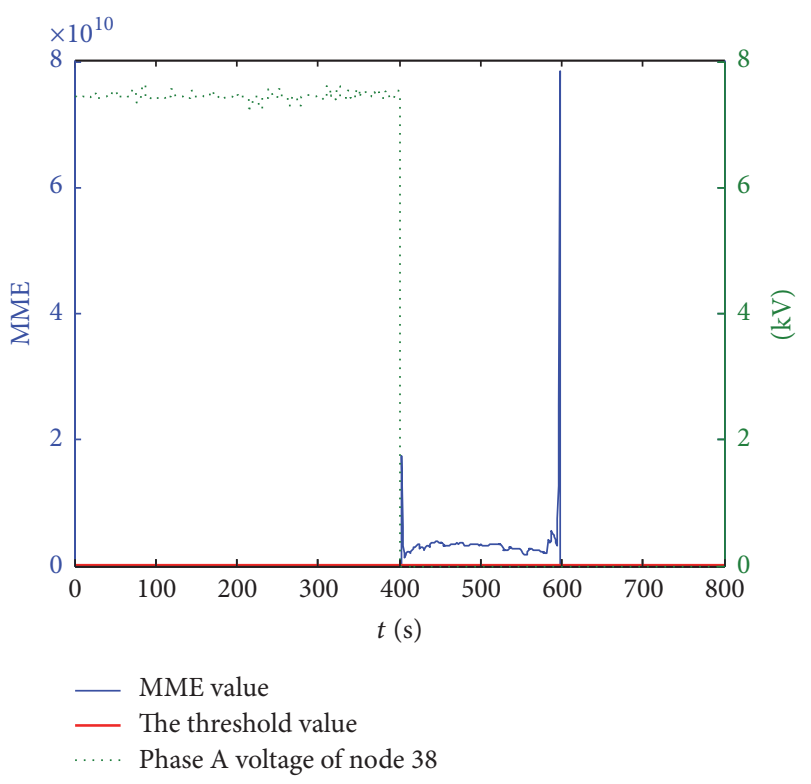

FIGURE 7: Abnormal state detection of three-phase short circuit.

in the faulty place and its downstream branch drops to 0 , and little change occurs in other branches.

According to the physical phenomena in the case of disconnection failure, the simulation data scenario is as follows. It is assumed that if a disconnection fault occurs in phase A at branch 26_38 between nodes 26 and 38, the circuit breaker between nodes 25 and 26 does not work and, in the 38_39,39_40, and 40_41 branch, phase A branch power dropped to 0 after failure.

A branch power matrix was used as the pending matrix for the sliding window sample. The branch power matrix of the system could be expressed as follows:

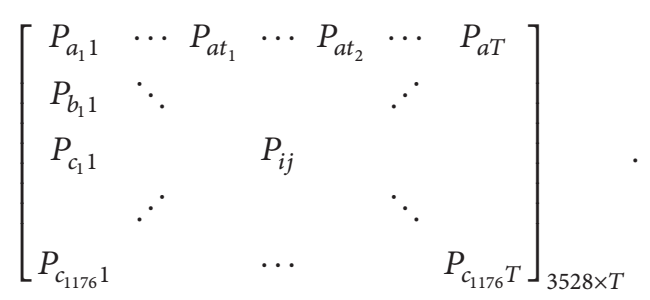

In (6), $P_{a_{1}}, P_{b_{1} 1}$, and $P_{c_{1} 1}$ represent the three-phase branch power value of node 1 in the first moment, and $P_{i j}$ shows onephase branch power value of a node at moment $j$.

At $300 \mathrm{~s}$, the fault occurs in branch 26_38 (Figure 8). In this case, the MME value rises sharply above the threshold value, and the curve shows a spike. The MME curve is different from that obtained by single-phase grounding and threephase short-circuit anomaly detection. The curve shows only a spike that is caused by the different types of matrix data. Power is not as stable as the voltage fluctuates more than in the normal situation. When the sliding window is sliding, the last row of normal data that leaves the window matrix has little effect on the data correlation change in the matrix, so that the MME curve of the power matrix appears only as a spike when an exception occurs.

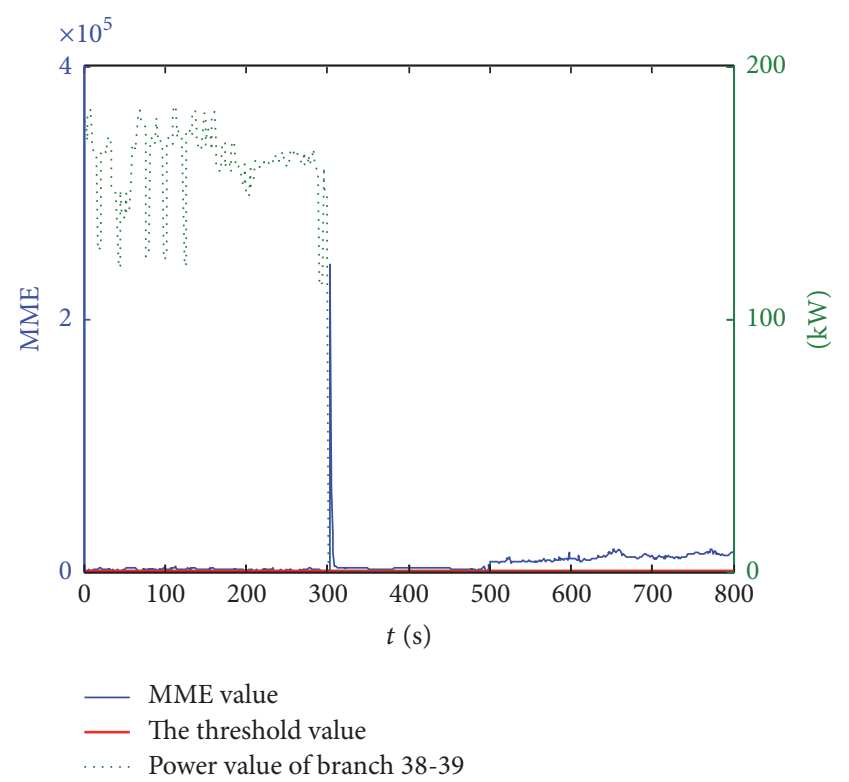

FIGURE 8: Abnormal state detection of a disconnect situation.

4.4. Abnormal State Identification under Load Mutation. In the case of a sudden change in load, when the power of a node rises abruptly, the effect of the other nodes is very small because of the stability of the power system.

According to the physical phenomena in the case of load mutation anomaly, the simulation data scenario was as follows: for node 38, an abnormality of "load jump and dump" was set. That is, at node 38 , the power was either reduced to zero or increased three times. The node power matrix of the system could be expressed as follows:

$$
\left[\begin{array}{ccccccc}
P_{a_{1} 1} & \cdots & P_{a_{1} t_{1}} & \cdots & P_{a_{1} t_{2}} & \cdots & P_{a_{1} T} \\
P_{b_{1} 1} & \ddots & & & \ddots & \\
P_{c_{1} 1} & & P_{i j} & & \\
& \ddots & & \ddots & \\
P_{c_{1177}} & & & \cdots & & P_{c_{1177} T}
\end{array}\right]_{3531 \times T}
$$

In (7), $P_{a_{1} 1}, P_{b_{1} 1}$, and $P_{c_{1} 1}$ represent the node power value of node 1 in the first moment, and $P_{i j}$ shows the one-phase power value of a node at moment $j$.

As shown in Figure 9, the load drop occurred at node 38 at $350 \mathrm{~s}$, and the MME curve spiked at random, after which, the MME curve fluctuated randomly for some time. In Figure 10, at $400 \mathrm{~s}$, the load on node 38 rose suddenly, and the MME curve showed a spike similar to the load dump. The time of occurrence of the abnormality can be detected by detecting the peak of the MME curve.

\section{Conclusions}

(1) This paper presents a method for detecting an abnormal state of running system based on a stochastic matrix theory and uses the sliding window to construct the sampling matrix 


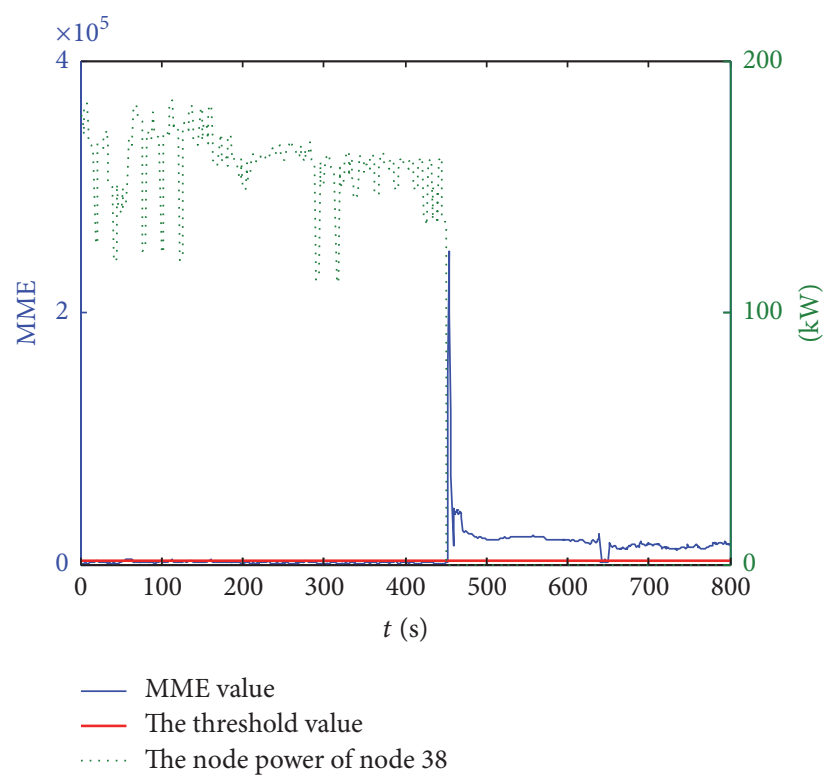

FIGURE 9: Abnormal state detection of load rising.

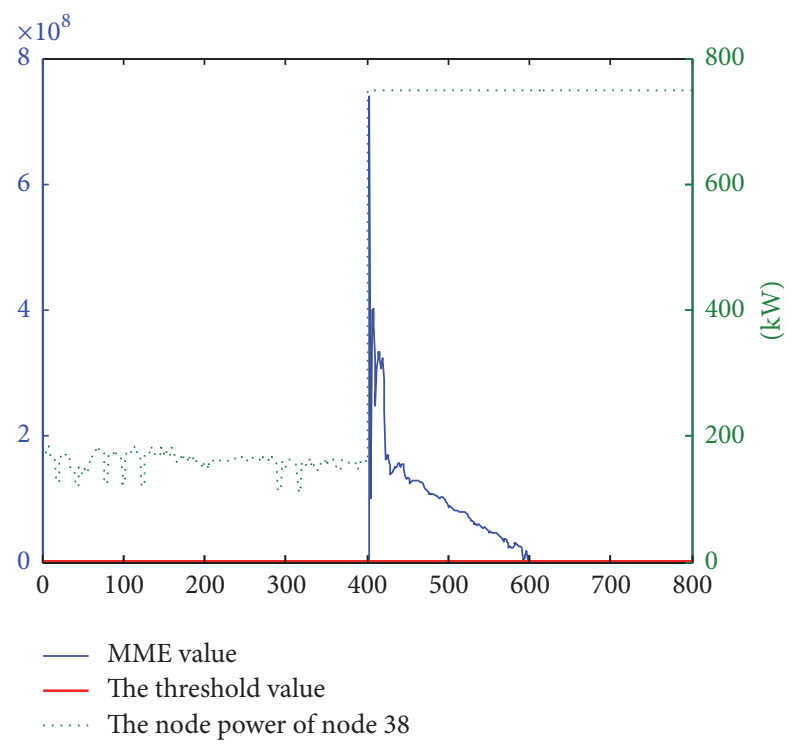

Figure 10: Abnormal state detection of load rising.

for real-time analysis. Historical and current status data are fully utilized. In this paper, the data sampling of each period is analyzed continuously in the running history, and the running state of the system is reflected in the MME curve.

(2) Compared with the traditional method in a running state analysis, this method, which emerged out of the physical model based on the RMT, greatly have better adaptability.

(3) The experimental results of four anomaly cases can be used to detect an anomaly occurrence time based on the MME method without depending on the physical model and fault type of the grid, which proves the effectiveness of the proposed method.

\section{Conflicts of Interest}

The authors declare that they have no conflicts of interest.

\section{Acknowledgments}

Special thanks are extended to the Key Technology Project of the State Grid Corporation of China (EPRIPDJK[2015]1495).

\section{References}

[1] S. Chen, L. Dong, and W. Yun, "Operation situational awareness based on dynamic power flow for a profound analysis of active distribution network," in Proceedings of the CIRED Workshop 2016, IET.

[2] L. Gong, J. Yang, L. Zhang, G. Li, M. Cai, and B. Dong, "Operational status recognition based on FCM and ANFIS for distribution network," Dianli Zidonghua Shebei/Electric Power Automation Equipment, vol. 36, no. 4, pp. 85-92, 2016 (Chinese).

[3] M. Asari, Y. Nakano, N. Ito et al., "Method of inferring operation status of distributed generation systems in distribution section," in Proceedings of the 1st IEEE-PES/IAS Conference on Sustainable Alternative Energy, (SAE), IEEE, September 2009.

[4] T. Zhao, Y. Zhang, and D. Zhang, "Application technology of big data in smart distribution grid and its prospect analysis," Power System Technology, vol. 38, no. 12, pp. 3305-3312, 2014 (Chinese).

[5] Z. Dongxia, X. Miao, L. Liu et al., "Research on development strategy for smart grid big data," Proceedings of the CSEE, vol. 35, no. 1, pp. 2-12, 2015 (Chinese).

[6] X. He, Q. Ai, R. C. Qiu, W. Huang, L. Piao, and H. Liu, "A big data architecture design for smart grids based on random matrix theory," IEEE Transactions on Smart Grid, vol. PP, no. 99, 2015.

[7] Y. Song, "Present status and challenges of big data processing in smart grid," Power System Technology, vol. 37, no. 4, pp. 927-935, 2013 (Chinese).

[8] R. C. Qiu, Z. Hu, Z. Chen et al., "Cognitive radio network for the smart grid: experimental system architecture, control algorithms, security, and microgrid testbed," IEEE Transactions on Smart Grid, vol. 2, no. 4, pp. 724-740, 2011.

[9] T. Huang, "Online distributed security feature selection based on big data in power system operation," Automation of Electric Power Systems, vol. 40, no. 4, pp. 137-140, 2016 (Chinese).

[10] J. Gantz and D. Reinsel, "Extracting value from chaos," IDC iView, pp. 1-12, 2011.

[11] GTM Research, "The soft grid 2013-2020: Big data \& utility analytics for smart grid-research excerpt [R/OL]," Tech. Rep., GTM, 2013, http://www.giiresearch.com/report/gm257044soft-grid-big-data-utility-analytics-smart-grid.html.

[12] B. Cova, N. Losignore, P. Marannino, and M. Montagna, "Contingency constrained optimal reactive power flow procedures for voltage control in planning and operation," IEEE Transactions on Power Systems, vol. 10, no. 2, pp. 602-608, 1995.

[13] M. Liu, S. K. Tso, and Y. Cheng, "An extended nonlinear primaldual interior-point algorithm for reactive-power optimization of large-scale power systems with discrete control variables," IEEE Transactions on Power Systems, vol. 17, no. 4, pp. 982-991, 2002.

[14] S. Feng, Y. Chen, and F. Gao, "Signal detection based on maximum-minimum eigenvalue in rician fading channel," in IEEE International Conference on Advanced Infocomm Technology, 2012. 
[15] A. Ahmed, Y. F. Hu, J. M. Noras, P. Pillai, R. A. Abd-Alhameed, and A. Smith, "Random matrix theory based spectrum sensing for cognitive radio networks," in Proceedings of the Internet Technologies and Applications (ITA), IEEE, Wrexham, UK, September 2015.

[16] Y. Zeng and Y. Liang, "Maximum-minimum eigenvalue detection for cognitive radio," in Proceedings of the 18th Annual IEEE International Symposium on Personal, Indoor and Mobile Radio Communications (PIMRC'07), September 2007.

[17] Y. Zeng, C. L. Koh, and Y.-C. Liang, "Maximum eigenvalue detection: theory and application," in Proceedings of the IEEE International Conference on Communications (ICC '08), pp. 4160-4164, Beijing, China, May 2008. 


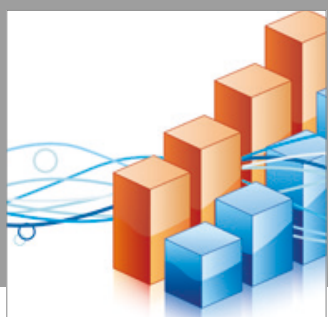

Advances in

Operations Research

vatersals

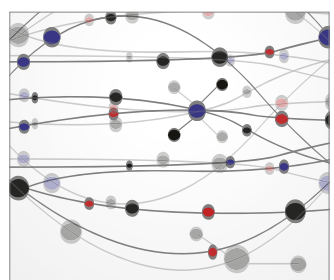

\section{The Scientific} World Journal
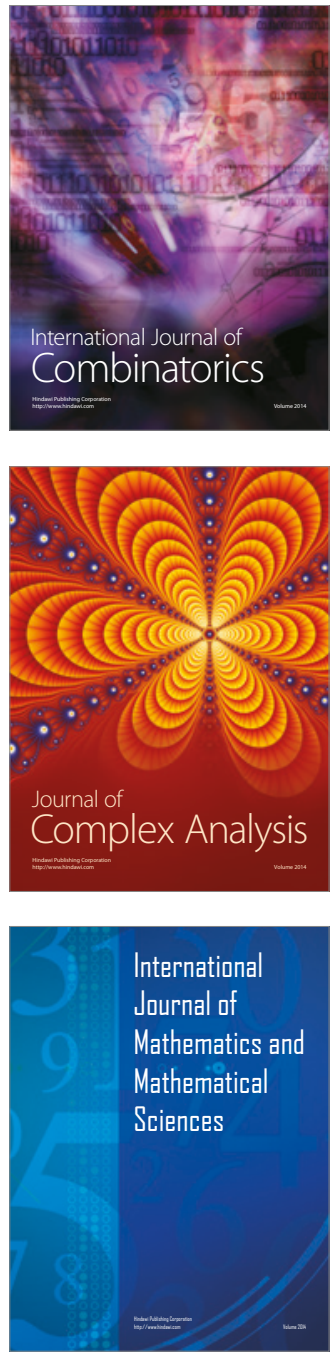
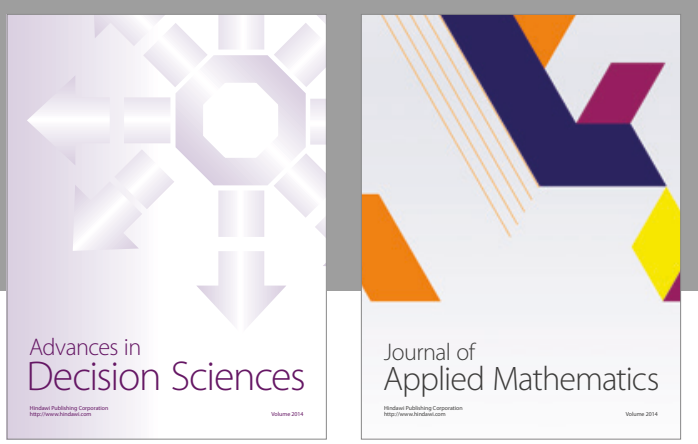

Algebra

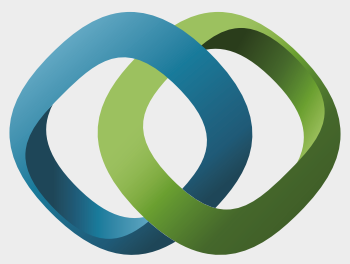

\section{Hindawi}

Submit your manuscripts at

https://www.hindawi.com
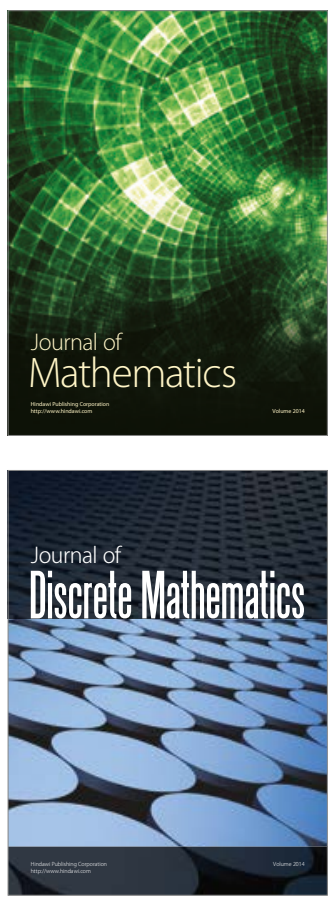

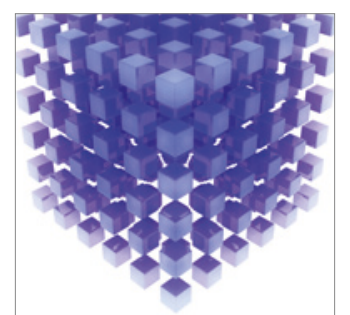

Mathematical Problems in Engineering
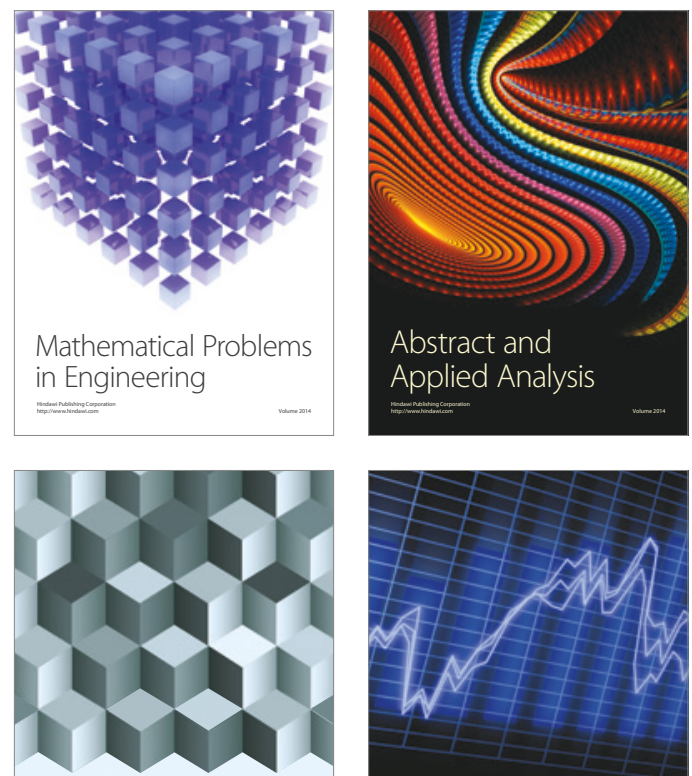

Journal of

Function Spaces

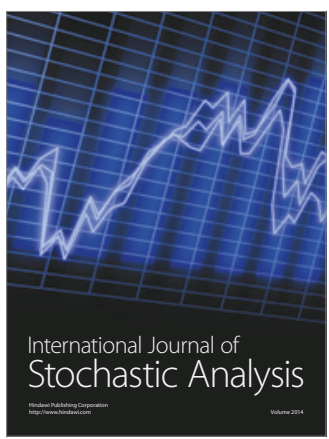

Probability and Statistics
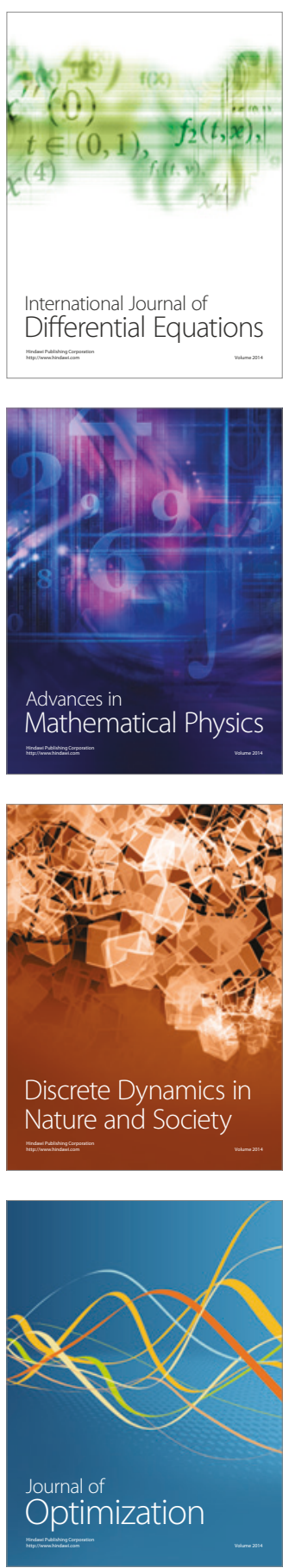